\title{
HNF1B expression regulates ECI2 gene expression, potentially serving a role in prostate cancer progression
}

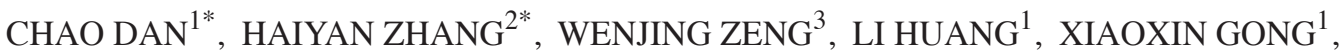 \\ HAO LI ${ }^{1}$, ERJIANG YANG ${ }^{1}$, LI WANG $^{1}$ and QISHENG YAO ${ }^{1}$ \\ Departments of ${ }^{1}$ Urology and Andrology, ${ }^{2}$ Blood Transfusion, and ${ }^{3}$ Anesthesiology, \\ Taihe Hospital, Hubei University of Medicine, Shiyan, Hubei 442000, P.R. China
}

Received November 21, 2017; Accepted September 28, 2018

DOI: $10.3892 / \mathrm{ol} .2018 .9677$

\begin{abstract}
Prostate cancer is the most common form of cancer in men, with increased incidence rates observed in older individuals. Prostate cancer is primarily driven via activation of the androgen receptor (AR), the principal transcriptional factor governing prostate cancer cellular programming and its associated metabolism. One of the downstream targets of AR is hepatocyte nuclear factor-1 $\beta$ (HNF1B), an important oncogenic transcription factor in prostate cancer. In the present study, the regulatory role of HNF1B in enoyl-CoA- $(\Delta)$ isomerase 2 (ECI2) expression in the transgenic adenocarcinoma of the mouse prostate (TRAMP) mouse model was investigated. Using this model, tumor progression and associated pathological alterations at 12,18 and 24 weeks were analyzed. Histological sectioning revealed pathological alterations over time, including thickening of glandular epithelial cells (12 weeks), increases in cellular proliferation (18 weeks), and extensive thickening and hardening of the tissue layer (24 weeks). Expression levels of HNF1B and ECI2 proteins were validated by immunohistochemistry and western blotting at different stages of prostate cancer development. HNF1B and ECI2 exhibited minimal differences in protein expression at 12 weeks in $\mathrm{TRAMP}^{+}$mice. However, by 18 weeks, $\mathrm{TRAMP}^{+}$mice exhibited multi-fold increases in HNF1B expression levels, along with downregulation of ECI2. These effects were reversed at 24 weeks, indicating an important time-dependent regulation of gene expression. Taken together, these results demonstrated that upon tumor progression, the
\end{abstract}

Correspondence to: Dr Qisheng Yao or Dr Li Wang, Department of Urology and Andrology, Taihe Hospital, Hubei University of Medicine, 32 South Renmin Road, Shiyan, Hubei 442000, P.R. China E-mail: oppenheimtacauh@yahoo.com

E-mail:wqn8so@163.com

*Contributed equally

Key words: prostate cancer, enoyl-CoA- $(\Delta)$ isomerase 2, transgenic adenocarcinoma of the mouse prostate model, hepatocyte nuclear factor $1 \beta$, androgen receptor initial tumor-protective effect of HNF1B is lost along with downregulated expression of HNF1B and increased expression of ECI2.

\section{Introduction}

The prostate gland is an accessory reproductive organ in males located between the bladder and penis. Cancer of the prostate is the most common type of cancer in the USA and was the second leading cause of cancer-associated mortality in 2016 (1). Uncontrolled growth associated with cellular migration and metastasis is the leading cause of cancer-associated mortality (2), with $17-34 \%$ of patients exhibiting metastatic disease at the time of initial diagnosis (3). Late-stage diagnosis remains an important problem, accounting for $37-43 \%$ of all cases, and is a significant risk factor for disease-associated mortality. Cancer screening tests, including screening for the prostate-specific antigen, have been useful for early detection, although the low sensitivity $(20.5 \%)$ and specificity $(93.6 \%)$ of this test highlight its limitations for prostate cancer detection (4).

Androgens and the androgen receptor (AR) are an important group of male steroid hormones that regulate prostate growth. Upon binding to androgen, the AR acts as a transcriptional factor, serving a major role in the proliferation, invasion and viability of prostate cancer cells (5). First-line therapies for prostate cancer directly target this pathway, by blocking androgen production or through suppression of the AR. However, despite the initial efficacy of these therapies, acquired resistance remains unavoidable (6). Subsequent lines of treatment, including chemotherapy and radiotherapy, may offer certain benefits, although typically these only extend life for an additional few months $(7,8)$. The scope for further advances in prostate cancer treatment remains limited due to mutations in numerous oncogenes, including phosphatase and tensin homolog, cellular tumor antigen p53, BRCA2 DNA repair associated, AR and MYC proto oncogene, which limit the effectiveness of targeted therapies (9-11). A greater understanding of the genes and mechanisms underlying prostate cancer progression is therefore necessary to develop more effective therapies.

Hepatocyte nuclear factor $1 \mathrm{~b}$ (HNF1B) is an important transcriptional factor that regulates embryonic survival and 
vertebrate development (12). Recently, it has been demonstrated that increased HNF1B expression may help protect against prostate cancer (13). By contrast, overexpression of genes such as enoyl-CoA-( $\Delta$ ) isomerase 2 (ECI2), an important regulator of fatty acid metabolism, may promote prostate cancer growth (14), although the complex interaction between these two genes remains poorly understood. In the present study, the functional association between HNF1B and ECI2 in promoting prostate cancer was analyzed.

\section{Materials and methods}

Mouse model of prostate cancer. Heterozygous male and female mice at 5 weeks old [C57BL/6-Tg $8247 \mathrm{Ng} / \mathrm{J}$ transgenic adenocarcinoma of the mouse prostate (TRAMP)] were purchased (Jackson Laboratory, Bar Harbor, ME, USA) and allowed to breed to obtain homozygous males. The males were bred with female FVB/NJ mice (Jackson Laboratory) to obtain C57BL/6 TRAMP x FVB mice, as described previously (15). TRAMP mice develop a progressive form of prostate cancer. Male mice at 6 weeks old (C57BL/6 TRAMP x FVB; $n=18)$, divided equally into three groups $(n=6)$, were used in all experiments. The mice were fed an AIN-93G diet and provided water ad libitum. The housing conditions in which the mice were maintained were $24-26^{\circ} \mathrm{C}, 55-60 \%$ humidity and a light/dark cycle of $14 / 10 \mathrm{~h}$. Mice without TRAMP overexpression were used as controls. Mice were sacrificed at 12, 18 and 24 weeks, and the prostate glands were dissected and subjected to further analysis. All the animals that were used in the present study, and the protocol followed, were approved by the institutional ethical committee (Taihe Hospital, Hubei University of Medicine, Shiyan, China).

Histological imaging. Dissected prostate glands were cut into small pieces and fixed in $10 \%$ formalin solution for $48 \mathrm{~h}$ at $40^{\circ} \mathrm{C}$. Tissues were subjected to gradual dehydration by transfer into increasing concentrations of isopropyl alcohol and embedded in paraffin blocks. Using a microtome, tissue sections were cut into $5 \mu \mathrm{m}$ thick slices and mounted on glass slides. Mounted sections were stained with hematoxylin (5-7 min) and counterstained with eosin (30 sec) at room temperature to visualize the cellular nucleus and cytoplasm. Final mounting was performed using DPX solution, which hardens to permanently seal the section on the slide. The samples were visualized under a light microscope at x20 magnification.

Immunohistochemistry. Tissue sections were cut and mounted onto glass slides, as described above. Cellular endogenous peroxide activity was blocked by incubating the sections in $3 \% \mathrm{H}_{2} \mathrm{O}_{2}$ solution in methanol. Slides were transferred into antigen retrieval solution $(10 \mathrm{mM}$ citrate buffer; $\mathrm{pH}$ 6.0) and incubated at $95^{\circ} \mathrm{C}$ for $7 \mathrm{~min}$ to unmask the antigenic epitope. Bovine serum albumin (BSA; 4\%; Sigma-Aldrich; Merck KGaA, Darmstadt, Germany) was used as a blocking buffer ( $2 \mathrm{~h}$ at room temperature) to reduce non-specific binding of antibodies. Primary antibodies against HNF1B (cat. no. CL0374; Abnova, Taipei, Taiwan; 1:200 dilution) or ECI2 (cat. no. 201243; Creative Diagnostics, New York, NY, USA; 1:250 dilution) were diluted in $1 \%$ BSA solution in $1 \mathrm{X}$ PBS and poured over the sections. Following treatment, slides were incubated at $4^{\circ} \mathrm{C}$ overnight, washed three times with $1 \mathrm{X}$ PBS, and incubated with the anti-mouse or anti-rabbit horseradish peroxidase (HRP)-conjugated secondary antibody (cat. nos. ab6789 or ab6721, respectively; 1:4,000 or 1:3,500 dilution, respectively; both Abcam, Cambridge, MA, USA) at room temperature for $1 \mathrm{~h}$. Slides were washed again in $1 \mathrm{X}$ PBS and developed with 3,3'-diaminobenzidine solution at room temperature for $10 \mathrm{~min}$. The signals obtained were visualized under a light microscope at x20 magnification.

Western blotting. Dissected prostate gland tissues were weighed and cut into small species. Tissue were homogenized with $2 \mathrm{X}$ protein sample buffer [4\% SDS, $20 \%$ glycerol, $0.004 \%$ bromophenol blue, $0.125 \mathrm{M}$ Tris/ $\mathrm{HCl}(\mathrm{pH} 6.8), 10 \%$ 2-mercaptoethanol] and diluted with water to a final $1 \mathrm{X}$ working concentration. The prepared samples were heated in a boiling water bath for 10 min along with sample buffer, which helps to preserve protein expression. The protein concentration was quantified using the Lowry method and $70 \mu \mathrm{g}$ was loaded into each well. The protein samples were separated by SDS-PAGE on a $12 \%$ gel, at $50 \mathrm{~V}$ for $3 \mathrm{~h}$, transferred to a polyvinylidene difluoride membrane, and blocked in 3\% BSA in TBS with Tween-20 at room temperature for $1 \mathrm{~h}$. The membrane was probed with anti-HNF1B antibody (cat. no. CL0374; Abnova; 1:300 dilution), anti-ECI2 antibody (cat. no. 201243; Creative Diagnostics; 1:500 dilution) or anti- $\beta$-actin antibody (cat. no. ab8227; Abcam; 1:1,000 dilution) overnight at $4^{\circ} \mathrm{C}$. Following washing three times with $1 \mathrm{X}$ PBS with Tween 20, the membrane was incubated with a specific HRP-conjugated secondary antibody (anti-mouse IgG HRP; Abcam; cat. no. ab6789; 1:5,000 dilution) or (anti-rabbit IgG HRP; Abcam; cat. no. ab6721; 1:4,000 dilution) at room temperature for $2 \mathrm{~h}$. Following incubation, the membrane was washed with 1X PBST and developed using a diaminobenzidine substrate kit (Abcam; cat. no. ab64238). HNF1B and EC12 expression levels were normalized to $\beta$-actin expression levels. The band intensities were measured using ImageJ software (version 1.5; https://imagej.net/ImageJ).

Statistical analysis. Statistical analyses for the obtained data were performed using SPSS for Windows (version 11.0; SPSS, Inc., Chicago, IL, USA). All the experiments were performed independently in triplicate and the results are expressed as the mean \pm standard error of the mean. The statistical significance was assessed using analysis of variance followed by Tukey's post hoc test for multiple comparisons. $\mathrm{P}<0.05$ was considered to indicate a statistically significant difference.

\section{Results}

Mice that express TRAMP show progressive prostate cancer. Prostate cancer progression was assessed in C57BL/6 TRAMP $\mathrm{x}$ FVB, a transgenic mouse line with enhanced susceptibility to prostate cancer due to TRAMP overexpression. Pathological outcomes were assessed at 12, 18 and 24 weeks, and were determined based on histological analysis of the prostate gland (Fig. 1). Prostates from 12-week-old control mice revealed a multi-lobed structure with a minimal glandular epithelium, 
A

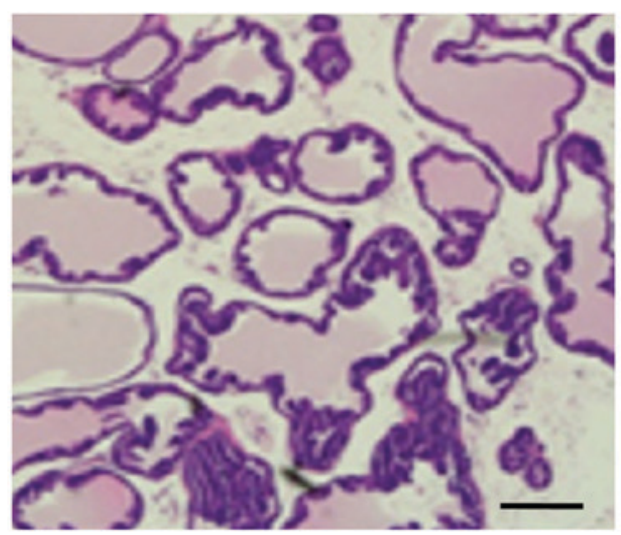

C

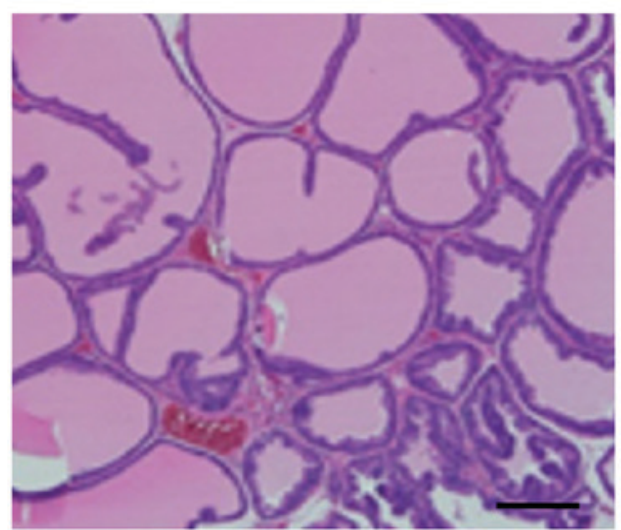

E

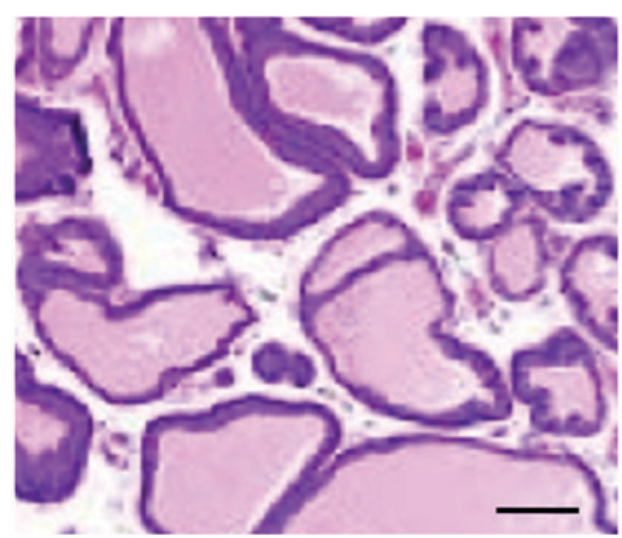

B

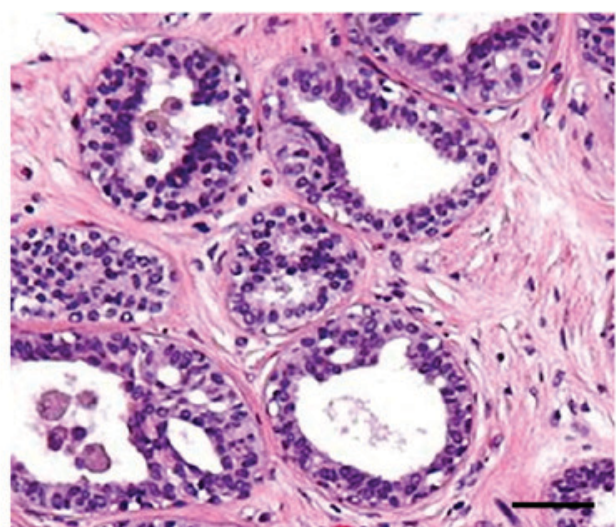

D

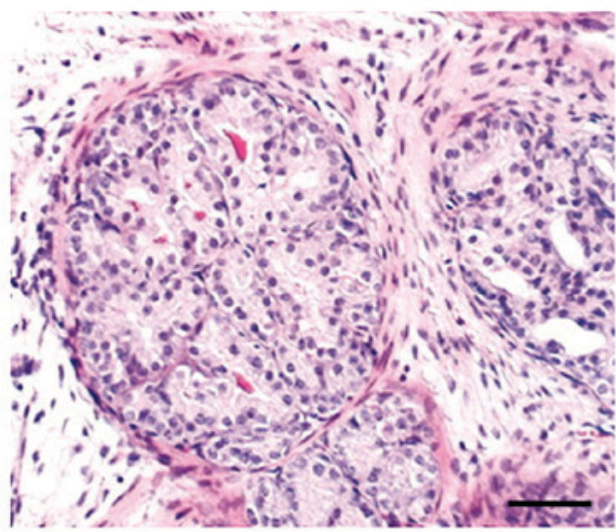

$\mathrm{F}$

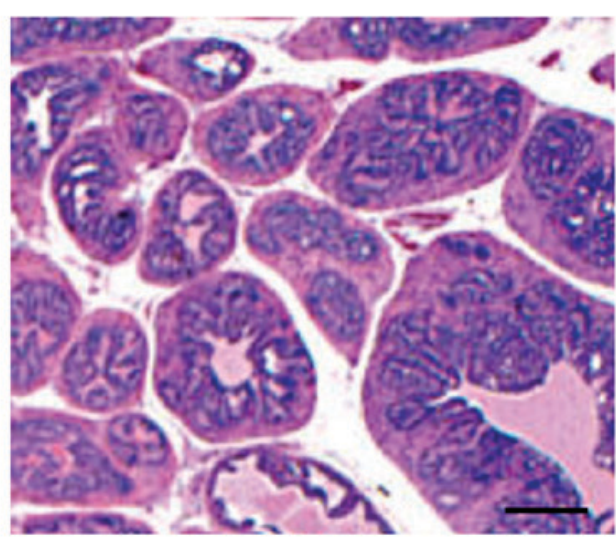

Figure 1. Tumor progression and pathological alterations in histological sections. (A) Prostate tissue from 12-week-old control mice displaying various lobular structures. (B) By 12 weeks, TRAMP ${ }^{+}$mice presented glandular epithelial thickening. (C) Development of fatty tissues between the lobular structures of control mice at 18 weeks. (D) Initial cell proliferative phase in 18 -week-old TRAMP ${ }^{+}$mice. (E) Control mice at 24 weeks displayed greater glandular epithelial thickening with minimal hyperplasia. (F) The 24 -week-old TRAMP ${ }^{+}$mice displayed extensive glandular epithelial thickening, along with hardening of the tissue layers. Scale bar, $50 \mu \mathrm{m}$. TRAMP, transgenic adenocarcinoma of the mouse prostate.

and regular spacing between lobes (Fig. 1A). By contrast, 12-week-old TRAMP ${ }^{+}$mice exhibited an initial thickening of the glandular epithelium with marked proliferation between the lobes (Fig. 1B). Development of fatty tissue between prostate lobes was evident by week 18 in control mice (Fig. 1C), compared with increased cellular infiltration in TRAMP ${ }^{+}$ mice with migration towards the interior of the lobes (Fig. 1D). By week 24, control mice displayed a gradual thickening of epithelial cells with minimal hyperplasia (Fig. 1E), whereas $\mathrm{TRAMP}^{+}$mice exhibited a progressive hardening of the lobes with extensive thickening of glandular cells and increased hyperplasia (Fig. 1F).
Expression of HNF1B with prostate cancer progress. $\mathrm{HNF} 1 \mathrm{~B}$ is a transcriptional factor that regulates a variety of important cellular processes $(16,17)$, including responses to hormones and growth factors. In the present study, the expression levels of HNF1B in different grades of prostate cancer histological sections were assessed (Fig. 2). HNF1B displayed minimal expression levels in control and TRAMP tissues (Fig. 2A and C) at 12 weeks. However, at week 18, TRAMP $^{+}$mice exhibited HNF1B expression in $\sim 90 \%$ of cells (Fig. 2E), consistent with the greater degree of cellular proliferation observed in these mice. By contrast, the more advanced tumors observed in 24-week-old $\mathrm{TRAMP}^{+}$mice 

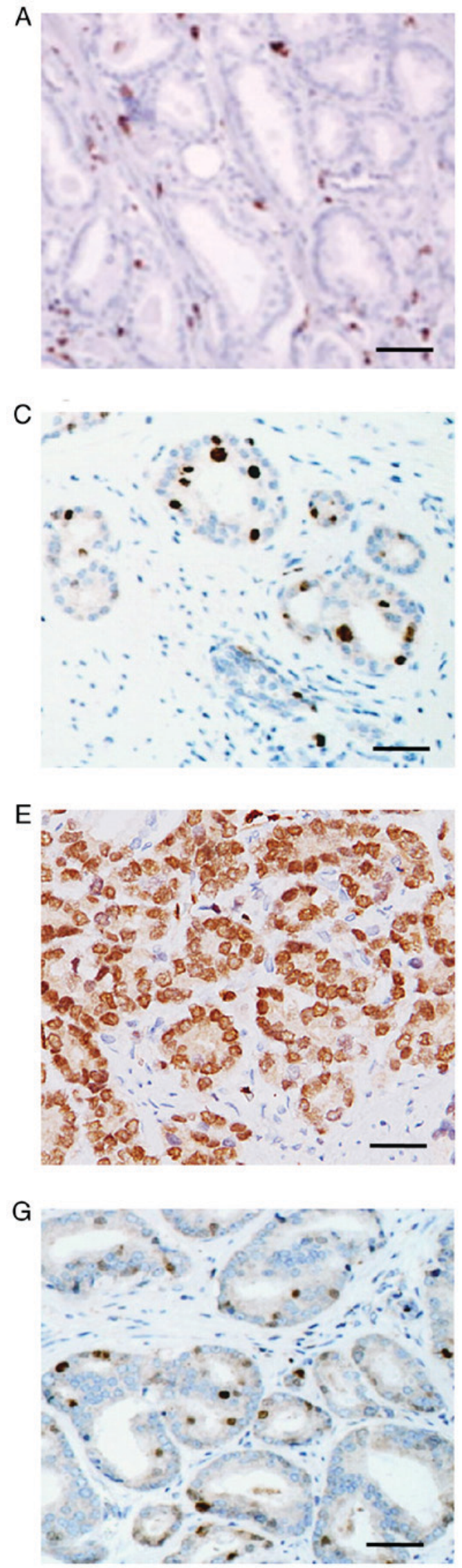

B

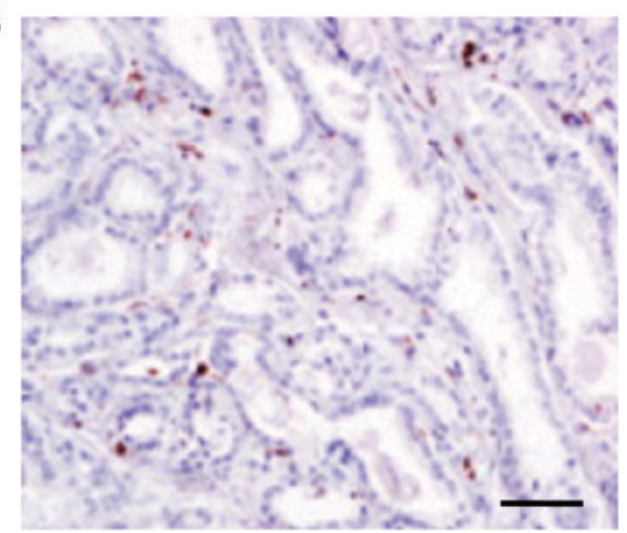

D

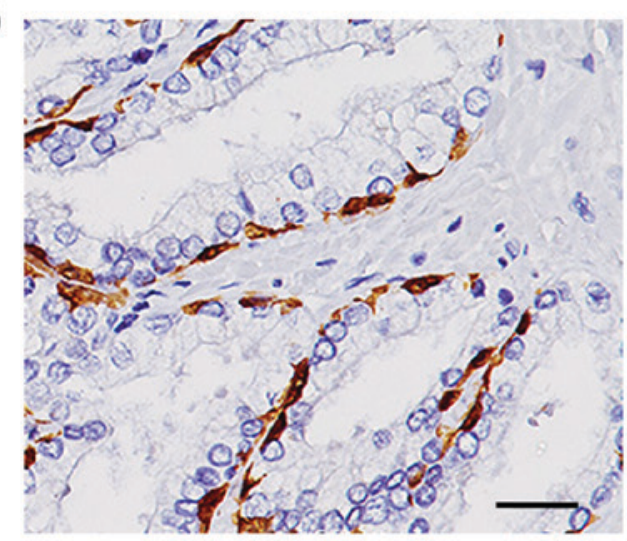

$\mathrm{F}$

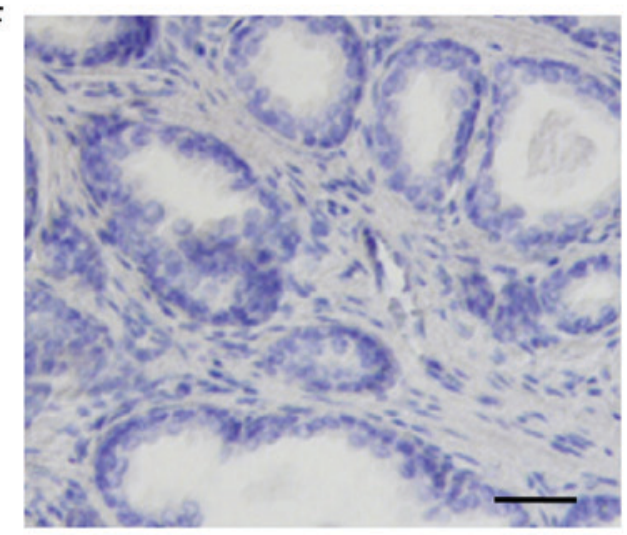

$\mathrm{H}$

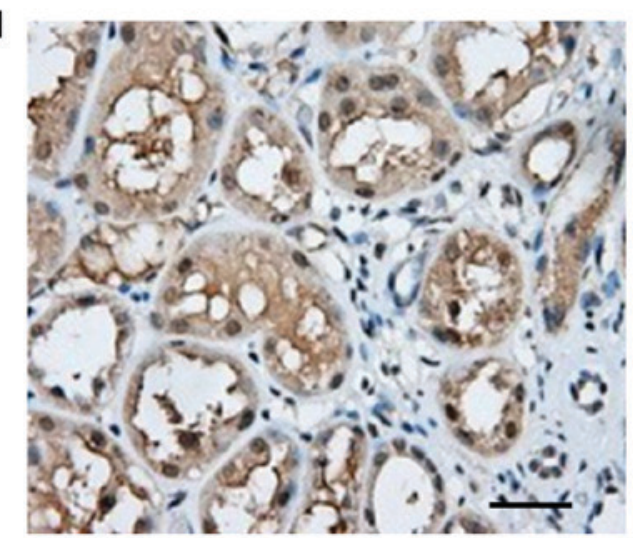

Figure 2. Controlled expression of ECI2 by HNF1B. (A) Control prostate tissue sample with minimal expression of HNF1B. (B) Control prostate tissue displaying

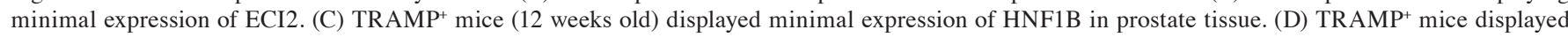
minimal ECI2 expression in prostate tissue at 12 weeks. (E) TRAMP ${ }^{+}$mice (18 weeks old) displayed overexpression of HNF1B in prostate glandular tissue. (F) At 18 weeks, TRAMP ${ }^{+}$mice displayed downregulated expression of ECI2 in the initial proliferative tissue. (G) TRAMP ${ }^{+}$mice (24 weeks old) displayed downregulated expression of HNF1B in hardened prostate tissues. (H) At 18 weeks, TRAMP ${ }^{+}$mice displayed overexpression of ECI2 in hardened prostate tissue. Scale bar, $50 \mu \mathrm{m}$. ECI2, enoyl-CoA- $(\Delta)$ isomerase 2; HNF1B, hepatocyte nuclear factor-1- $\beta$; TRAMP, transgenic adenocarcinoma of the mouse prostate. 

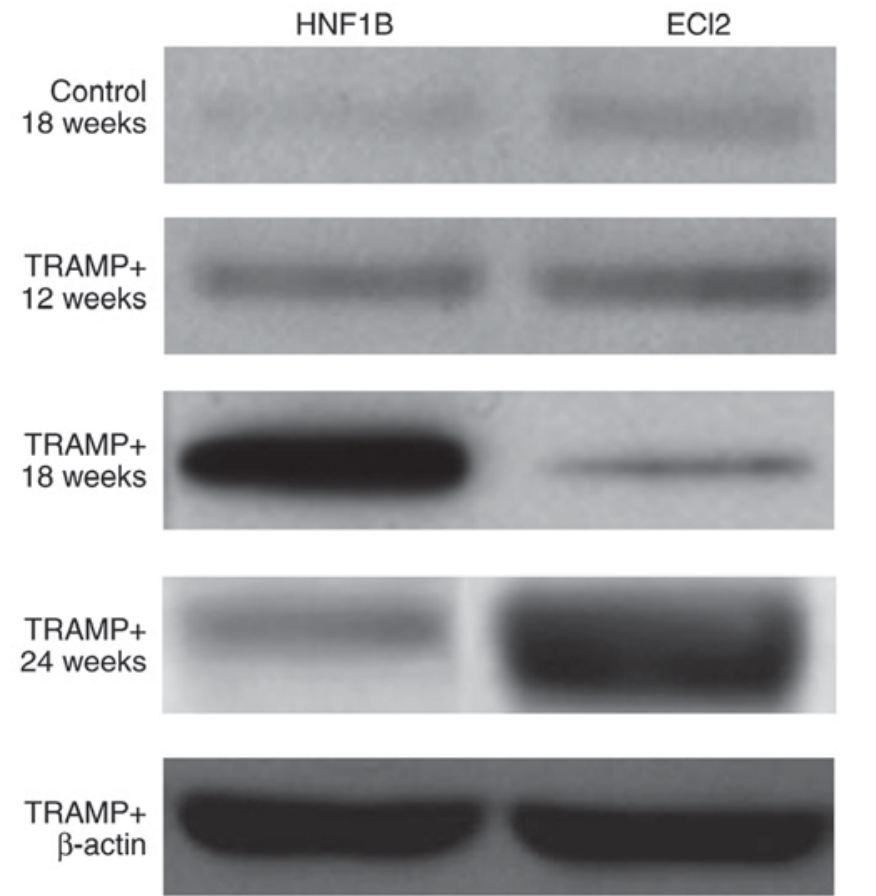

Figure 3. Western blot analysis of HNF1B and ECI2 expression. Lane 1: Expression levels of HNF1B in controls and 12-(initial stage), 18-(proliferative stage) and 24-week-old TRAMP ${ }^{+}$mice. Lane 2: Expression levels of ECI2 in controls and 12-, 18-, and 24-week-old TRAMP ${ }^{+}$mice. The loading control was $\beta$-actin. ECI2, enoyl-CoA- $(\Delta)$ isomerase 2 ; HNF1B, hepatocyte nuclear factor-1- $\beta$; TRAMP, transgenic adenocarcinoma of the mouse prostate.

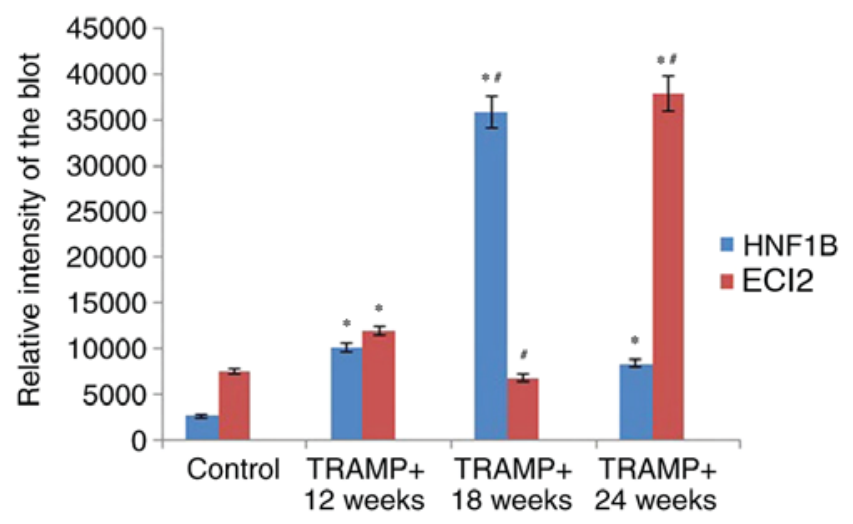

Figure 4. Expression study of HNF1B and ECI2 during prostate cancer progression. Quantitative expression of HNF1B, ECI2 and $\beta$-actin in control, 12, 18 and 24-week-old TRAMP ${ }^{+}$mice. The results were obtained from three independent experiments and are presented as the mean \pm standard deviation. The statistical analysis between the two variables was performed using analysis of variance followed by Tukey's post hoc test for multiple comparisons. Results are normalized to $\beta$-actin expression levels, and $\mathrm{P}<0.05$ was considered to indicate a statistically significant difference. ${ }^{*} \mathrm{P}<0.05$ vs. control and ${ }^{\text {"}} \mathrm{P}<0.05$ vs. TRAMP+12 weeks. ECI2, enoyl-CoA- $(\Delta)$ isomerase 2 ; HNF1B, hepatocyte nuclear factor-1 $\beta$; TRAMP, transgenic adenocarcinoma of the mouse prostate.

were characterized by decreases in HNF1B expression levels, indicative of important alterations in gene regulation in vivo (Fig. 2G).

Altered ECI2 expression in response to HNF1B. Given the importance of transcription factors, including HNF1B, the role of HNF1B gene expression on other downstream targets, including ECI2, was assessed. ECI2 exhibited minimal alterations in gene expression levels in control mice at 12 weeks (Fig. 2B), compared with mild overexpression in TRAMP $^{+}$mice (Fig. 2D). Notably, 18-week-old TRAMP ${ }^{+}$ mice exhibited marked downregulation of ECI2 expression levels (Fig. 2F) compared with 12-week-old TRAMP ${ }^{+}$mice (Fig. 2B); however, this trend did not persist, with prominent overexpression of ECI2 evident as the tumor progressed further (Fig. 2H).

Protective effect of HNF1B through regulation of ECI2 in the initial proliferative phase of prostate cancer. Given the differential expression levels observed between HNF1B and ECI2, western blotting was used to confirm the alterations in protein expression levels in $\mathrm{TRAMP}^{+}$mice over time. The patterns of protein expression observed for HNF1B and ECI2, as revealed by western blotting (Figs. 3 and 4), were similar to those revealed by immunohistochemistry (Fig. 2). The level of HNF1B expression in prostate tissue was reported to be 3.55 -fold increased in 18 -week-old TRAMP $^{+}$mice compared with 12 -week-old TRAMP $^{+}$mice, and it subsequently became downregulated (by a factor of 0.17 ) in 24-week-old $\mathrm{TRAMP}^{+}$mice compared with 12-week-old TRAMP ${ }^{+}$mice (Fig. 4). The results for ECI2 demonstrate that its expression was 0.44-fold downregulated in 18-week-old TRAMP ${ }^{+}$mice compared with 12 -week-old $\mathrm{TRAMP}^{+}$mice, and its expression pattern indicated 3.17-fold overexpression in 24-week-old TRAMP ${ }^{+}$mice compared with 12-week-old TRAMP ${ }^{+}$mice (Fig. 4). These results suggested that ECI2 may be regulated by HNF1B in TRAMP ${ }^{+}$mice at 18 and 24 weeks in the TRAMP model of prostate cancer (Figs. 3 and 4).

\section{Discussion}

Prostate cancer is the most common form of cancer in men, with the incidence being higher among older men $(18,19)$. In the present study, the TRAMP mouse model of prostate cancer was used in order to better assess various aspects of disease progression in vivo (20). TRAMP mice were demonstrated to develop various pathological features with increasing age, characterized by potential neoplasia in the early stages of disease (12 weeks), followed by remodeling of epithelial structures at week 18 (21). As the tumor progressed, lobes present in the inner tissues of the prostate began to harden, although the mechanisms underlying these alterations remain poorly understood (22).

HNF1B is a transcriptional factor that serves an important role in organelle development and tumorigenesis (23) and has direct connections to at least 12 cancer-associated genes, including nuclear receptor subfamily 4 group A member 1, BCL2-associated athanogene 1 and phosphatidylinositol-4,5-bisphosphate 3-kinase catalytic subunit- $\gamma$. Furthermore, this gene has been reported to serve a role in prostate cancer by modulating androgenic hormone signaling (24), making it a good candidate for further study. It was demonstrated that HNF1B expression levels were associated with ECI2 expression levels, which has important implications for future studies. However, in the present study, the expression levels of HNF1B 
and ECI2 exhibited opposing trends in 18- and 24-week-old $\mathrm{TRAMP}^{+}$mice. Previous studies have suggested mechanisms by which ECI2 may be regulated by AR (25); however, the precise mechanisms regulating this gene, and its association with HNF1B, remain elusive. In the present study it was demonstrated that the initial proliferative time point for prostate cancer (18 weeks) was characterized by increased expression levels of HNF1B, conferring a potential protective effect against tumor development.

Taken together, the data presented demonstrate that the TRAMP model is optimal for studying prostate cancer progression. The analyses revealed baseline alterations between initial proliferative cells, thickening of epithelial cells and hardening of tissues. It was also reported that the increased expression levels of HNF1B in initial stages of the disease serve a tumor-protective role, although upon tumor progression its expression is downregulated and expression levels of ECI2 are upregulated.

\section{Acknowledgements}

Not applicable.

\section{Funding}

The present study was funded by Taihe Hospital (Shiyan, China), the Scientific and Technological Project of Shiyan City of Hubei Province (grant no. 17Y13) and the Natural Science Foundation of Hubei Province of China (grant no. 2013CFC070).

\section{Availability of data and materials}

All data generated or analyzed during this study are included in this published article.

\section{Authors' contributions}

$\mathrm{CD}$ and $\mathrm{HZ}$ conducted a literature search, designed experiments, analyzed data and wrote the manuscript. CD, HZ and WZ performed experiments. $\mathrm{LH}$ and $\mathrm{XG}$ interpreted the data and contributed to the experimental work. HL, EY and LW performed minor experiments and critically revised the manuscript. QY designed experiments, provided critical feedback and obtained funding.

\section{Ethical approval and consent to participate}

The animals used in the present study, and the protocols followed, were approved by the institutional ethical committee at Taihe Hospital, Hubei University of Medicine (Shiyan, China).

\section{Patient consent for publication}

Not applicable.

\section{Competing interests}

The authors declare that they have no competing interests.

\section{References}

1. Munkley J, Vodak D, Livermore KE, James K, Wilson BT, Knight B, Mccullagh P, Mcgrath J, Crundwell M, Harries LW, et al: Glycosylation is an androgen-regulated process essential for prostate cancer cell viability. EBioMedicine 8: 103-116, 2016

2. Hanahan D and Weinberg RA: Hallmarks of cancer: The next generation. Cell 144: 646-674, 2011.

3. Munkley J, McClurg UL, Livermore KE, Ehrmann I, Knight B, Mccullagh P, Mcgrath J, Crundwell M, Harries LW, Leung HY, et al: The cancer-associated cell migration protein TSPAN1 is under control of androgens and its upregulation increases prostate cancer cell migration. Sci Rep 7: 5249, 2017.

4. Ankerst DP and Thompson IM: Sensitivity and specificity of prostate-specific antigen for prostate cancer detection with high rates of biopsy verification. Arch Ital Urol Androl 78: 125-129, 2006.

5. Snoek R, Cheng H, Margiotti K, Wafa LA, Wong CA, Wong EC, Fazli L, Nelson CC, Gleave ME and Rennie PS: In vivo knockdown of the androgen receptor results in growth inhibition and regression of well-established, castration-resistant prostate tumors. Clin Cancer Res 15: 39-47, 2009.

6. Nelson WG, De Marzo AM and Isaacs WB: Prostate cancer. N Engl J Med 349: 366-381, 2003.

7. De Bono JS, Oudard S, Ozguroglu M, Hansen S, Machiels JP, Kocak I, Gravis G, Bodrogi I, Mackenzie MJ, Shen L, et al: Prednisone plus cabazitaxel or mitoxantrone for metastatic castration-resistant prostate cancer progressing after docetaxel treatment: A randomised open-label trial. Lancet 376: 1147-1154, 2010.

8. Parker C, Nilsson S, Heinrich D, Helle SI, O'Sullivan JM, Fosså SD, Chodacki A, Wiechno P, Logue J, Seke M, et al: Alpha emitter radium-223 and survival in metastatic prostate cancer. $\mathrm{N}$ Engl J Med 369: 213-223, 2013.

9. Grasso CS, Wu YM, Robinson DR, Cao X, Dhanasekaran SM, Khan AP, Quist MJ, Jing X, Lonigro RJ, Brenner JC, et al: The mutational landscape of lethal castrate resistant prostate cancer. Nature 487: 239-243, 2012.

10. Robinson D, Van Allen EM, Wu YM, Schultz N, Lonigro RJ, Mosquera JM, Montgomery B, Taplin ME, Pritchard CC, Attard G, et al: Integrative clinical genomics of advanced prostate cancer. Cell 161: 1215-1228, 2015.

11. Kumar A, White TA, MacKenzie AP, Clegg N, Lee C, Dumpit RF, Coleman I, Ng SB, Salipante SJ, Rieder MJ, et al: Exome sequencing identifies a spectrum of mutation frequencies in advanced and lethal prostate cancers. Proc Natl Acad Sci USA 108: 17087-17092, 2011.

12. Hiesberger T, Shao X, Gourley E, Reimann A, Pontoglio M and Igarashi P: Role of the hepatocyte nuclear factor-1beta (HNF-1beta) C-terminal domain in Pkhd1 (ARPKD) gene transcription and renal cystogenesis. J Biol Chem 280: 10578-10586, 2005.

13. Ross-Adams H, Ball S, Lawrenson K, Halim S, Russell R, Wells C, Strand SH, Ørntoft TF, Larson M, Armasu S, et al: HNF1B variants associate with promoter methylation and regulate gene networks activated in prostate and ovarian cancer. Oncotarget 7: 74734-74746, 2016.

14. Itkonen HM, Brown M, Urbanucci A, Tredwell G, Ho Lau C, Barfeld S, Hart C, Guldvik IJ, Takhar M, Heemers HV, et al: Lipid degradation promotes prostate cancer cell survival. Oncotarget 8: 38264-38275, 2017.

15. Opoku-Acheampong AB, Unis D, Henningson JN, Beck AP and Lindshield BL: Preventive and therapeutic efficacy of finasteride and dutasteride in TRAMP mice. PLoS One 8: e77738, 2013.

16. Senkel S, Lucas B, Klein-Hitpass L and Ryffel GU: Identification of target genes of the transcription factor HNF1beta and HNFlalpha in a human embryonic kidney cell line. Biochim Biophys Acta 1731: 179-190, 2005.

17. Stevens VL, Ahn J, Sun J, Jacobs EJ, Moore SC, Patel AV, Berndt SI, Albanes D and Hayes RB: HNF1B and JAZF1 genes, diabetes, and prostate cancer risk. Prostate 70: 601-607, 2010.

18. Itkonen HM, Engedal N, Babaie E, Luhr M, Guldvik IJ, Minner S, Hohloch J, Tsourlakis MC, Schlomm T and Mills IG: UAP1 is overexpressed in prostate cancer and is protective against inhibitors of N-linked glycosylation. Oncogene 34: 3744-3750, 2015.

19. Itkonen HM, Minner S, Guldvik IJ, Sandmann MJ, Tsourlakis MC, Berge V, Svindland A, Schlomm T and Mills IG: O-GlcNAc transferase integrates metabolic pathways to regulate the stability of c-MYC in human prostate cancer cells. Cancer Res 73: 5277-5287, 2013. 
20. Gelman IH: How the TRAMP model revolutionized the study of prostate cancer progression. Cancer Res 76: 6137-6139, 2016.

21. Gingrich JR, Barrios RJ, Morton RA, Boyce BF, DeMayo FJ, Finegold MJ, Angelopoulou R, Rosen JM and Greenberg NM: Metastatic prostate cancer in a transgenic mouse. Cancer Res 56: 4096-4102, 1996.

22. Wei L, Wang J, Lampert E, Schlanger S, DePriest AD, Hu Q, Gomez EC, Murakam M, Glenn ST, Conroy J, et al: Intratumoral and intertumoral genomic heterogeneity of multifocal localized prostate cancer impacts molecular classifications and genomic prognosticators. Eur Urol 71: 183-192, 2017.

23. Yu DD, Guo SW, Jing YY, Dong YL and Wei LX: A review on hepatocyte nuclear factor-1beta and tumor. Cell Biosci 5: 58, 2015.
24. Hu YL, Zhong D, Pang F, Ning QY, Zhang YY, Li G, Wu JZ and Mo ZN: HNF1b is involved in prostate cancer risk via modulating androgenic hormone effects and coordination with other genes. Genet Mol Res 12: 1327-1335, 2013.

25. Mallik I, Davila M, Tapia T, Schanen B and Chakrabarti R: Androgen regulates $\mathrm{Cdc} 6$ transcription through interactions between androgen receptor and $\mathrm{E} 2 \mathrm{~F}$ transcription factor in prostate cancer cells. Biochim Biophys Acta 1783: 1737-1744, 2008.

(i) This work is licensed under a Creative Commons Attribution-NonCommercial-NoDerivatives 4.0 International (CC BY-NC-ND 4.0) License. 